\title{
The Solar Ultraviolet Magnetograph Investigation Sounding Rocket Program
}

\author{
E. A. West, K. Kobayashi, J. M. Davis and G. A. Gary \\ NASA/NSSTC, 320 Sparkman Drive, Huntsville, AL 35805
}

\begin{abstract}
This paper will describe the objectives of the Marshall Space Flight Center (MSFC) Solar Ultraviolet Magnetograph Investigation (SUMI) and the unique optical components that have been developed to meet those objectives. A sounding rocket payload has been developed to test the feasibility of magnetic field measurements in the Sun's transition region. The optics have been optimized for simultaneous measurements of two magnetic sensitive lines formed in the transition region (CIV at $1550 \AA$ and MgII at $2800 \AA$ ). This paper will concentrate on the polarization properties SUMI's toroidal varied-line-space (TVLS) gratings and its system level testing as we prepare to launch in the Summer of 2008.
\end{abstract}

Keywords: UV polarization, Solar Magnetograph, Sun, Sounding Rocket, Toroidal gratings, VLS gratings, double Wollaston polarizer

\section{INTRODUCTION}

The Solar Ultraviolet Magnetograph Investigation, SUMI, has been reported in several papers since this program began in $2000^{8,9,10}$. The emphasis in this paper will be to reference and summarize the test results presented in those papers while documenting the new tests of the last component that has been developed for SUMI, the toroidal variable-linespace (TVLS) gratings. Along with describing the results of the TVLS gratings, this paper will describe the issues associated with making system level tests of the SUMI payload that would be difficult if given the resources of a spacebased mission but are very challenging with the limited budget of a sounding rocket program.

Section 2 will review the scientific goals of SUMI and why this mission is important in understanding the 3D structure of the magnetic field on the Sun. The flight hardware that makes up the SUMI payload is described in Section 3 with emphasis on the TVLS gratings. Section 4 will emphasize the testing of the SUMI payload which is too large to fit into the NSSTC test facility ${ }^{12}$ which measured all of SUMI's optics. Because of this a custom vacuum ultraviolet (VUV) collimator had to be developed that was within the budget constraints of a sounding rocket program while providing the data required to verify that SUMI could achieve the spatial, wavelength and polarization resolution necessary to meet its design goals. The purpose of this facility and its limitations as a calibration tool will be discussed. Section 5 will summarize the current mission goals of SUMI based on our expected FY08 funding.

\section{SCIENCE GOALS}

This section will describe the importance of direct magnetic field measurements at various heights in the solar atmosphere and how the SUMI sounding rocket program plans to verify that the transition region measurements are possible. While the SUMI sounding rocket program is a proof-of-concept mission, the goal of this program is to obtain high resolution CIV spectro-polarimetry data of a simple large sunspot so that this data can be compared to the theoretical models and NLTE radiative codes that are being developed for this important transition region line $\mathrm{e}^{1,2}$. While a large simple sunspot may not produce the polarization maps required to understand complicated structures in the transition region, SUMI's first flight is an essential step in understanding the CIV measurements and the modeling require to connect the photospheric magnetic field to the magnetic fields observed in the transition region. 


\subsection{Magnetic field measurements in the transition region}

The problem of how energy, stored in the solar magnetic field, is released to heat the corona and drive the dynamic phenomena of the outer atmosphere, flares and coronal mass ejections, remains unsolved. Neither theoretical or numerical models nor current observations are able to provide a conclusive story. We have learned that a substantial fraction of this energy propagates outward into the interplanetary medium in the form of radiation, the solar wind, energetic particles, and shocks that create, control and modify the structure of the interplanetary medium. These products constitute space weather and have been shown to affect the immediate terrestrial environment, to create hazards for terrestrial and space assets, and will, in the future, be a major concern for astronauts traveling through interplanetary space and working on the lunar surface. The solar input is the logical starting point for any model that describes and predicts space weather. Therefore understanding the processes that contribute to both the steady and transient release of magnetic energy into the solar atmosphere is a crucial element of these studies.

To understand the fundamental processes that lead to the explosive release of magnetic energy in solar flares and coronal mass ejections (CMEs), it is necessary to observe and infer the topology of the magnetic field and how it evolves prior to and during the energy release process. . Some success has been achieved in the characterization of the full vector field in the photosphere, where $\beta$, the ratio of the gas pressure to the magnetic pressure, is $>1$. At higher levels in the atmosphere (Figure 1$)^{3}$ where $\beta<1$, the magnetic field, through the Lorentz force, controls the structure and dynamics of the solar atmosphere, and rapid changes in its structure can produce energetic events. However, observations of the magnetic field at these higher levels have proven to be difficult, placing a serious limitation on our understanding of the physical processes actually occurring there.

The crucial and missing element is the ability to understand and describe quantitatively the transition layer where the field is no longer pressuredominated as in the photosphere. The need to understand the energy release processes was identified two decades $\mathrm{ago}^{4}$, and was reaffirmed as the major conclusion of a workshop on the definition of the scientific goals for "Beyond SolarB" (Moore, Davis, and Hathaway, 2001) ${ }^{5}$. To understand this process full vector magnetic field measurements at several different heights in the solar atmosphere, from the photosphere to the upper transition region where the field becomes force free, will be required. The vector field in the photosphere and low chromosphere has been measured with ground based instruments. Spacebased instruments such as the Michelson Doppler Interferometer (MDI) on SOHO have demonstrated

Table 1: This table shows a comparison of candidate lines that are being developed for current and future space-based photospheric, chromospheric and transition region vector magnetic field measurements.

\begin{tabular}{|c|c|c|c|c|c|}
\hline $\begin{array}{c}\text { Spectral } \\
\text { lines }\end{array}$ & $\begin{array}{c}\lambda \\
(\mathrm{nm})\end{array}$ & $\mathrm{g}$ & $\begin{array}{c}\lambda^{2} \times \mathrm{g} \\
\times 10^{4}\end{array}$ & $\begin{array}{c}\text { Height } \\
(\mathrm{km})\end{array}$ & Remarks \\
\hline \multicolumn{7}{|c|}{ Chromosphere } \\
\hline CIV & $\mathbf{1 5 5 . 0}$ & 1.2 & 2.9 & $\mathbf{2 2 0 0}$ & SUMI \\
\hline MgII & $\mathbf{2 8 0 . 0}$ & 1.2 & 9.4 & $\mathbf{2 0 0 0}$ & SUMI \\
\hline CaII & 854.2 & 1.1 & 80.2 & 1319 & \\
\hline NaI & 589.5 & 1.3 & 45.1 & 700 & \\
\hline \multicolumn{7}{|c|}{ Photosphere } \\
\hline FeI & 630.2 & 2.5 & 99.2 & 250 & Hinode \\
\hline
\end{tabular}

MTRAP $^{6}$ : The Magnetic Transition Region Probe has all of these lines in its baseline mission.

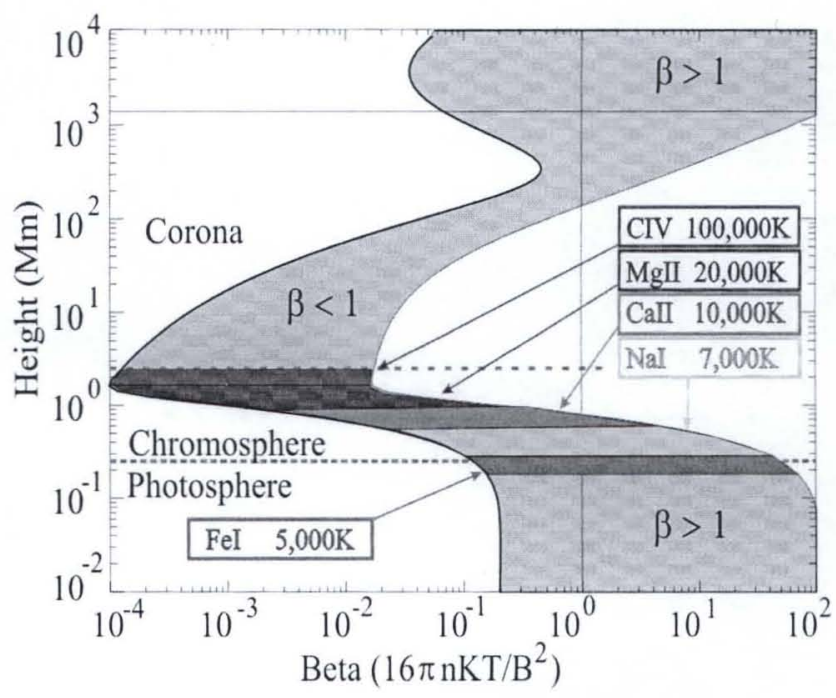

Figure 1. Ratio of gas pressure to magnetic pressure $(\beta)$ as a function of height, for an assumed range of photospheric magnetic field strength of 100 to $2500 \mathrm{G}$ (Garv, 2001) ${ }^{3}$. 
that continuous, distortion free, observations of the longitudinal magnetic field from space are very important in our understanding of the Sun's magnetic field and the SOT/Hinode and HMI/SDO missions ( 2008$)$ will extend our space based observations to include photospheric vector magnetic fields. Although these high resolution observations will greatly advance our knowledge of the behavior of the magnetic field in the photosphere, they will shed little light on how the magnetic field higher in the solar atmosphere transitions to a nearly force-free state. This requires measurement of the field using spectral lines that are formed at temperatures above $20,000^{\circ} \mathrm{K}$. The two spectral lines we have chosen for SUMI are MgII and CIV. These lines occur in the far ultraviolet and are inaccessible from the ground. Together with FeI, NaI and CaII, they form a quintet of magnetically sensitive lines chosen for a future mission called the Magnetic Transition Region Probe (MTRAP) ${ }^{6}$. The demonstration that the MgII and CIV lines can meet the requirements for the force free region is a prerequisite for this mission and is one of the objectives of the Solar Ultraviolet Magnetograph Investigation (SUMI).

\subsection{Goals for SUMI sounding rocket program}

By the early nineties it was apparent that vector field measurements in the upper chromosphere and transition region were needed, even though this was viewed as a difficult if not impossible task. The Ultraviolet Spectrometer and Polarimeter (UVSP) instrument on board the Solar Maximum Mission (SMM, 1980) was the first space-based instrument to try to map and understand polarization in the transition region. The UVSP observations of MgII suggested that this line was a potential candidate for the upper chromosphere? ${ }^{7}$. Although UVSP attempted measurements of the CIV line, photon statistics and the loss of the CIV line early in the mission failed to produce convincing evidence that this measurement was possible. While the scientific value of these observations has been acknowledged ${ }^{1,2}$, the measurements are difficult. The SUMI sounding rocket program was born from development programs ${ }^{8,9,10}$ to improve the overall wavelength and polarization efficiency for the CIV $(155 \mathrm{~nm})$ measurement while including the MgII measurements in its observing program. While these development programs were successful, accommodating the dualline instrument in a sounding rocket envelope has been challenging.

CIV has always been the driving force in the development of SUMI. While this line is formed primarily in the transition region which simplifies its interpretation, the magnetic sensitivity, $\lambda^{2} \mathrm{~g}$ where $\lambda$ is the wavelength and $\mathrm{g}$ is Lande $\mathrm{g}$ factor, is low when compared to lines in the visible and infrared wavelengths. Table 1 compares the SUMI lines with lines that are being used in future space-based telescopes. Certainly the results from SUMI will impact future missions as scientist try to understand the 3-D structure of the Sun's magnetic field. 


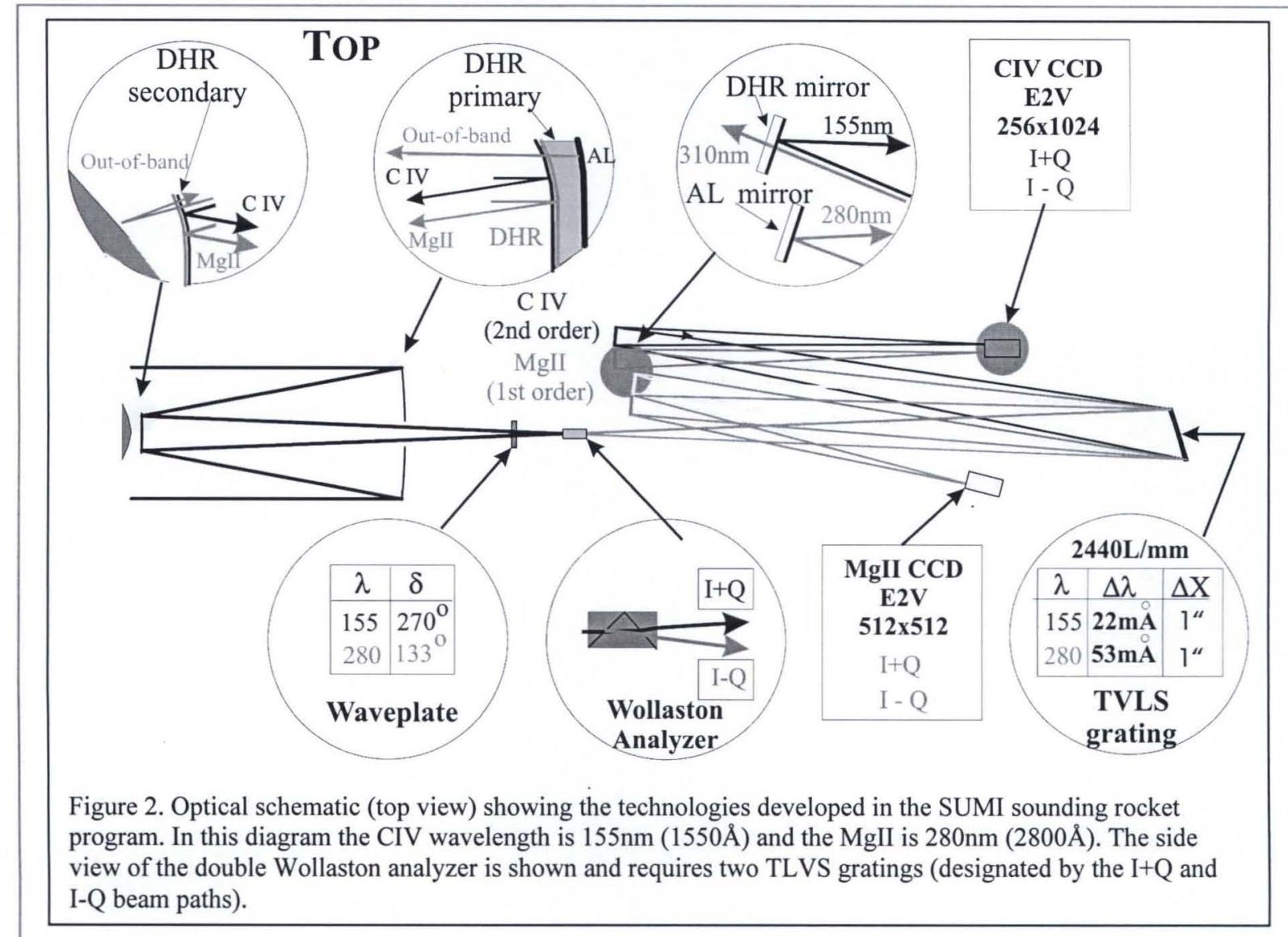

\section{DESCRIPTION OF SOUNDING ROCKET PAYLOAD}

The Solar Ultraviolet Magnetograph Investigation (SUMI) began as a set of development programs to improve the efficiency of polarization measurements in the ultraviolet. As demonstrated by Table 1, polarization measurements in the ultraviolet require high spectral resolution due to the small magnetic sensitivity $\left(\lambda^{2} \mathrm{~g}\right)$. Higher spectral resolution is required to "resolve" the Zeeman splitting. Therefore to reduce the length of the telescope while increasing the spectrograph, a cold mirror Ritchey-Chretien telescope was chosen over a traditional Gregorian telescope (§3.1). To improve the photon and polarization efficiency, a $\mathrm{MgF}_{2}$ double Wollaston polarizer was selected as the analyzer so that simultaneous measurements of orthogonal polarizations could be made. Ideally, full Stokes polarization measurements, [I, Q, U, V], should be made but photon statistics make linear polarization measurements (Q and U) impossible in CIV for a sounding rocket program. Therefore the waveplate is optimized for [I, Q, V] measurements in CIV and the full Stokes in MgII (\$3.2). Section 3.3 will describe the Toroidal Variable-Line-Space (TVLS) grating technology that is used to achieve the high spectral resolution while reducing the number of optics. The last section (\$3.4) will describe the camera systems and their interface to the SUMI onboard computers.

\subsection{Telescope optics}

A solar telescope design must solve the thermal problems associated with direct solar viewing. The simplest solution is a Gregorian telescope with a field stop between the primary and secondary mirrors. While this reduces the thermal load on the secondary by rejecting the unwanted light, the disadvantages to this approach are a limited field of view, a longer optical path, and a larger secondary for on-axis designs, which decreases the effective collecting area of the telescope. 
The SUMI approach for decreasing the thermal load on the secondary mirror is to use a RitcheyChretien telescope design with special dielectric coatings applied to the front surfaces of both the primary and secondary mirrors. These coatings reflect only the narrow wavelength ranges around the CIV and MgII emission lines which results in a "cold mirror", i.e., a "self-filtering" telescope. The rear surface of the primary mirror is figured and has an aluminum coating that reflects the unwanted radiation back through the telescope. The advantages of this design are that the field of view is not restricted (whole Sun could be imaged by a slitjaw camera) and, for a given instrument length, the smaller telescope size allows a larger spectrograph improving the wavelength resolution. These narrowband UV reflection coatings simplify the thermal environment, minimize infrared and visible light contamination of the spectral data, and act as a blocking filter for the spectrograph. The measured reflectance of these coatings is shown in Figure 3. Although the MgII bandpass may appear off center, its reflectivity was tuned to minimize the $1^{\text {st }}$ order reflectance of $3100 \AA$ into the $2^{\text {nd }}$ order CIV measurement.

\subsection{Polarimeter and slit jaw camera}

The first section following the telescope is the polarimeter, spectrograph slit and slit jaw camera. The polarimeter consists of a $\mathrm{MgF}_{2}$ waveplate and a double Wollaston analyzer. Due to the low photon flux at CIV and the weak linear polarization levels, the waveplate was designed to measure circular polarization at $\mathrm{CIV}\left(270^{\circ}\right.$ retardance at $1550 \AA$ ) and the full Stokes vector at MgII $\left(131^{\circ} \text { retardance at } 2800 \AA\right)^{9,11}$. During the flight SUMI will concentrate on circular polarization measurements when the payload is above $200 \mathrm{~km}$ (required height for CIV measurements) and will do the linear polarization measurements when the sounding rocket is below $200 \mathrm{~km}$.

The double Wollaston analyzer, which is a polarizing beamsplitter, is the most efficient UV polarizer in this wavelength band ${ }^{9}$. Figure 4 shows the orientation of the double Wollaston and the exiting linearly polarized beams. The orientation of the linear polarization and the polarization reflectivity of the TVLS gratings will be discussed in the next section (§3.3). The vertical linear polarization $(\mathrm{VP}=+\mathrm{Q})$ is defined to be the E field aligned to the slit (spatial axis), and the horizontal linear polarization $(\mathrm{HP}=-\mathrm{Q})$ is perpendicular to it (dispersion axis).

A dual-beam analyzer is very important in transition region magnetic field measurements since the CIV emission is a very dynamic. Without simultaneous measurements of orthogonal polarizations, intensity crosstalk could create false signals in the magnetic field measurements. Since both polarizations are observed, the total transmission is much higher than traditional reflective polarizers even at CIV which is near the cutoff wavelength of $\mathrm{MgF}_{2}(1150 \AA)$. Finally, the polarization resolution $\left(<10^{-3}\right)$ is higher and covers a larger wavelength range than traditional reflective analyzers which is very important for SUMI's CIV $(1550 \AA)$ and MgII $(2800 \AA)$ measurements. 
Table 2. Polarization reflectivity measurements for the six gratings fabricated by Jobin Yvon for SUMI: 2007 measurements. The gratings selected for the first flight are \#1 for the HP and \#3 for the VP.

\begin{tabular}{||c|c|c|c|c|c||}
\hline \multirow{2}{*}{ Grating number } & \multirow{2}{*}{ Coating } & \multicolumn{2}{|c|}{ Horizontal Polarization (HP) } & \multicolumn{2}{c|}{ Vertical Polarization (VP) } \\
\cline { 3 - 6 } & & CIV $\left(2^{\text {nd }}\right.$ order $)$ & MgII $\left(1^{\text {st }}\right.$ order $)$ & CIV $\left(2^{\text {nd }}\right.$ order $)$ & MgII $\left(1^{\text {st }}\right.$ order $)$ \\
\hline 1. CY11S* & AL/MgF2 & $12 \%$ & $39 \%$ & $29 \%$ & $52 \%$ \\
\hline 2. CY11O & AL/MgF2 & $11 \%$ & $33 \%$ & $15 \%$ & $38 \%$ \\
\hline 3. CY11N* & AL/MgF2 $(A L)$ & $12 \%(16 \%)$ & $41 \%(9 \%)$ & $30 \%(17 \%)$ & $54 \%(49 \%)$ \\
\hline 4. CY11P & AL & $16 \%$ & $11 \%$ & $18 \%$ & $52 \%$ \\
\hline 5. CY11R & AL & $12 \%$ & $15 \%$ & $20 \%$ & $62 \%$ \\
\hline 6. CY11M & AL & $14 \%$ & $16 \%$ & $17 \%$ & $61 \%$ \\
\hline
\end{tabular}

* Acton $\mathrm{AL} / \mathrm{MgF}_{2}$ coatings

For SUMI's first flight, the slit has a unique structure. The slit is on a $6 \mathrm{~mm}$ thick diamond turned aluminum substrate and is $\sim 3.7 \mathrm{~mm}$ long ( 3.5 arc minutes) with $0.5 \times 0.5 \mathrm{~mm}$ boxes on each end. The width of the slit has been split into two sections, a $30 \mu \mathrm{m}\left(\sim 1.6^{\prime \prime}\right)$ and a $60 \mu \mathrm{m}(\sim 3.2 ")$. Due to the exploratory nature of the SUMI measurements, the uncertainty in the target at launch and the limited observing time of the flight ( $\sim 6$ minutes), this structure was adopted to allow SUMI to capture enough photons to make meaningful polarization measurements at CIV.

The slitjaw camera has two functions. Initially, it is a "white-light" camera for real-time images to acquire the target. After the target is acquired, a filter wheel in front of the camera will be used to obtain narrowband context images of both the CIV and MgII lines. The slitjaw camera is the same E2V CCD used in the MgII measurements (§3.4). This $\mathrm{CCD}$ is a frame transfer, back-illuminated, uncoated silicon detector array with $512 \times 512$ pixels. The spatial resolution of each pixel has been set to $0.65^{\prime \prime}$ which limits the FOV of the slitjaw camera to5.6' x 5.6'.

TVLS grating 2 with $\mathrm{AL} / \mathrm{MgF}_{2}$

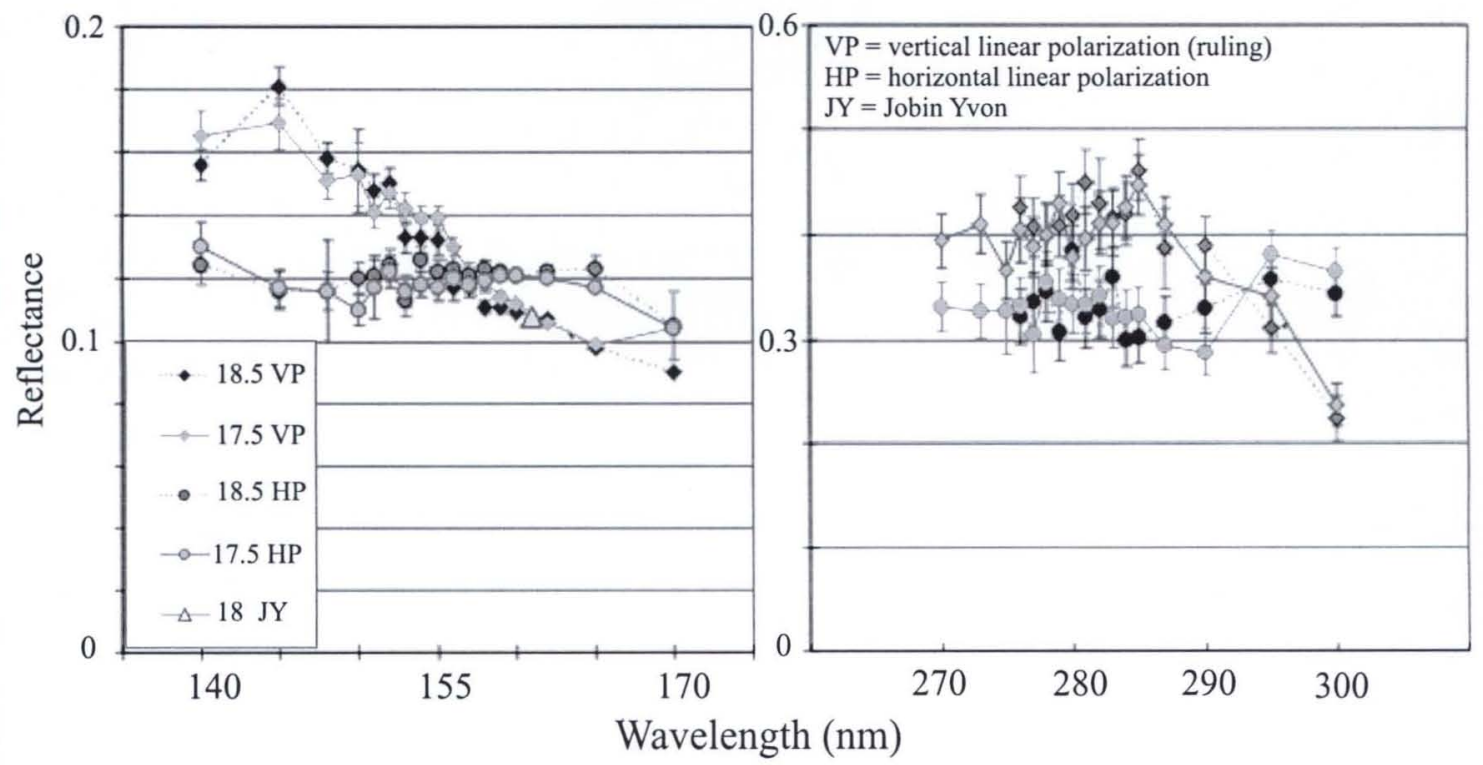

Figure 5. Polarization reflectivity measurements on TVLS grating with Aluminum- $\mathrm{MgF}_{2}$ coating covering SUMI's two wavelength bands 


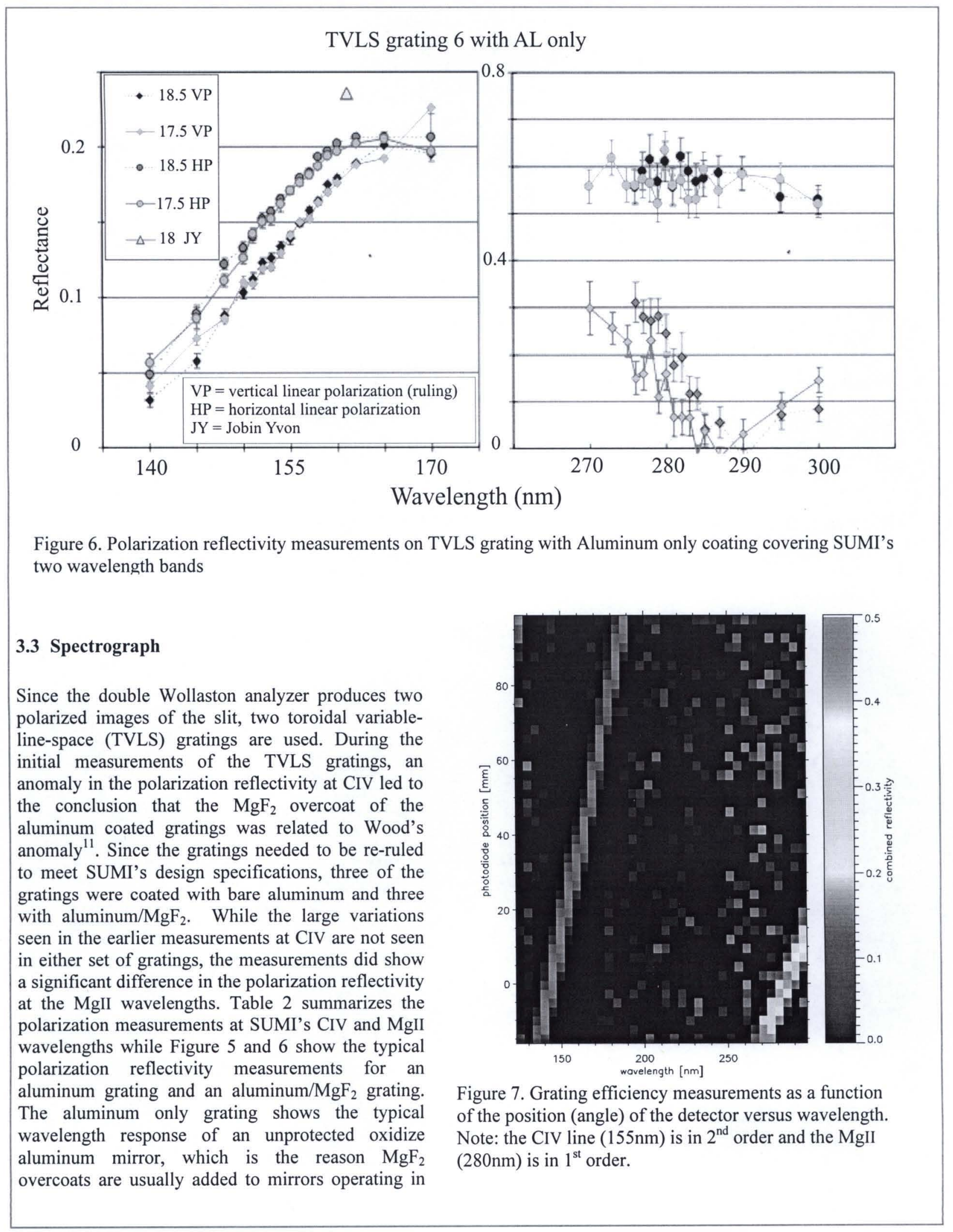


this wavelength region. For these gratings (Table 2) the difference between the oxidation loss of a bare aluminum and the aluminum $/ \mathrm{MgF}_{2}$ gratings is not significant at CIV. The surprising result is the large difference in the MgII polarization reflectivity which was not seen in our previous measurements ${ }^{11}$.

Sensitivity of the spectrograph gratings to the polarization exiting the double Wollaston has always been considered ${ }^{8}$. In our initial concept a quarterwave plate could be placed between the double Wollaston and gratings to convert the exiting linear polarization to circular, minimizing the difference in the reflectivity, such as that seen in Figure 6. While the quarterwave plate is a solution, it is also another "absorption loss" at CIV. Therefore, selecting a grating that has a high reflectivity for the horizontal polarization for MgII (Figure 5) and a second grating for the vertical polarization (Figure 6) simplifies the optical design and removes an optical element that will absorb CIV.

\subsection{UV cameras and SUMI data system}

The SUMI cameras are developed around the E2V back illuminated, bare silicon technology. This technology enhances the UV quantum efficiency but is currently limited to certain CCD structures since they not part of E2V's standard product line. For the slitjaw camera, and the MgII cameras (Figure 2) where high speed shuttering is required, a frame transfer $512 \times 512 \mathrm{CCD}$ array with $13 \mu \mathrm{m}$ pixels was selected. The CIV camera uses a full frame $1024 \times 256 \mathrm{CCD}$ array with $26 \mu \mathrm{m}$ pixels. To simplify the electrical wiring between the cameras and the data system, a USB 2.0 interface was selected. For ground-based testing and software development, a $\mathrm{MgF}_{2}$ window is mounted in front of all of the cameras. The measured quantum efficiency $(\mathrm{QE})$ for these cameras is $>60 \%$.

The SUMI sounding rocket has two data systems, designated the CIV and the MgII data systems, to control the four USB cameras, the rotating waveplate and to monitor the health and status of the SUMI payload. The data systems have a Dothan Pentium 738 low voltage processor running at $1.4 \mathrm{GHz}$ on a cPCI bus. All of the $\mathrm{cPCI}$ cards are conduction cooled, designed to survive a $12 \mathrm{G}$ launch and have an operating temperature range of -40 to $85^{\circ} \mathrm{C}$. Each data system has two USB and two Ethernet ports on the processor board. Since the CIV measurements have the highest priority, the CIV data system is the master and drives the timing and telemetry for the SUMI sounding rocket. The MgII data system is the slave and all of the data from the two MgII cameras is stored on the sounding rocket in a 4GB solid state disk drive. The slit jaw camera is connected to the second USB port on the CIV data system. During the ascent of the sounding rocket, the slitjaw camera has the highest priority for the telemetry downlink as the sounding rocket is pointed to its target. Any CIV images acquired during this time will be stored onto the CIV 4GB solid state disk. After the target is acquired, the CIV images will high the highest priority for the telemetry channel.

Originally the data systems were placed outside of the telescope/spectrograph structure but the need to push the center of gravity (CG) toward the telescope forced us to relocate them just aft of the primary mirror, above and below the spectrograph slit. This was done to minimize the weight that would have to be added to move the CG which would also require a heavier parachute for re-entry. Moving the data systems into the spectrograph structure adds some complexity but significantly increases the observing time ( 3 to 6 minutes).

\section{OPTICAL TESTING SUMI}

Since each optical element represents a loss in photons, especially at CIV, the concept for SUMI was to isolate the CIV and $\mathrm{MgII}$ lines using as few optics as possible. This approach led to the development of the dielectric high-reflectivity (DHR) coatings on the telescope mirrors (\$3.1, Figure 3) which act as a dual-line prefilter, an efficient beamsplitting double Wollaston analyzer (\$3.2) which doubles the efficiency, and TVLS gratings which provides both the wavelength resolution and imaging optics for the scientific cameras. While SUMI is very efficient in isolating our UV lines, traditional methods to align the instrument can not be used. Therefore a custom vacuum ultraviolet (VUV collimator) facility had to be developed that was within the budget constraints of a sounding rocket program while providing the data required to verify that SUMI could achieve the spatial, wavelength and polarization resolution necessary to meet its mission goals. While this facility can be used to align SUMI, it cannot produce the resolutions that SUMI can achieve. SUMI has higher wavelength resolution due to its 2440 lines/mm TVLS gratings versus the 1200 line/mm grating in the 


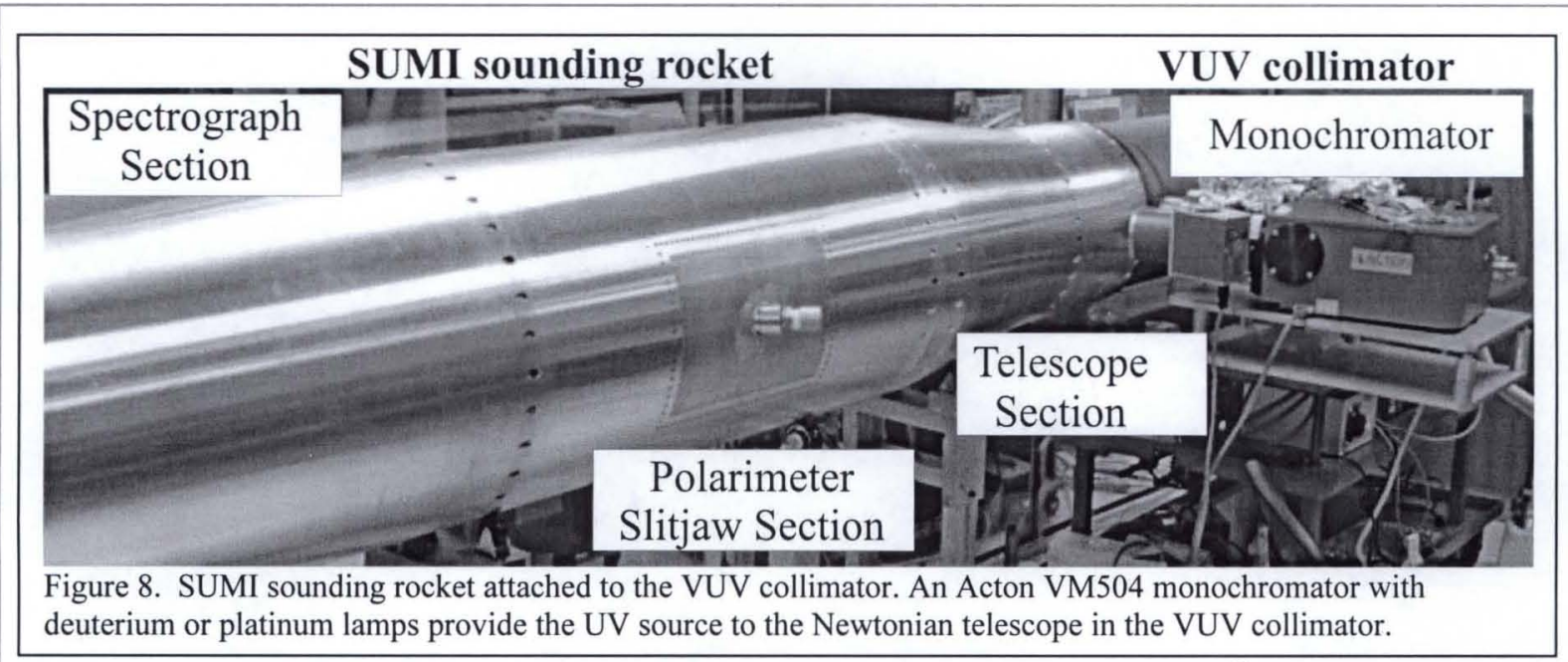

VUV monochromator, it has higher spatial resolution due to its custom VUV mirrors ${ }^{10}$ and higher F\#, and the polarization calibration must be achieved using the instrumental polarization produced by the VUV monochromator and collimating optics.

Although the VUV collimator cannot achieve the SUMI resolutions, it can be aligned with traditional instruments since it is an all reflecting system with aluminum $/ \mathrm{MgF}_{2}$ coatings. After aligning the VUV collimator, it can be used to provide the vacuum UV wavelengths needed to check the alignment of the SUMI telescope and spectrograph. This section will describe some of the test results that have been completed using the VUV collimator and the uncertainties in the alignment of SUMI.

\subsection{VUV collimator}

The VUV collimator consists of a Newtonian telescope and an Acton VM504 monochromator. Most of the optical problems with interfacing the VUV collimator with SUMI are related to the difference of the F\#'s of the Acton monochromator and SUMI. Originally an existing Cassegrain telescope was considered since it was a closer match (F\#) to SUMI but the photon loss between it and the monochromator was excessive and the "non-collimated" photons would become a large scatter light source. An off axis parabolic Newtonian system was considered but the cost of off axis mirrors, and the fact that the size of the secondary of the current Newtonian design could be made to match the secondary of SUMI, led to the development of the current VUV collimator (Figure 8).

\subsection{Wavelength and polarization tests}

While the VUV collimator will be used to align the SUMI telescope mirrors and estimate the spatial and polarization resolution, its primary function is to verify the location of the CIV and MgII lines on the spectrograph cameras. Due to the high spectral resolution required to resolve the Zeeman effect ( $22 \mathrm{~m} \AA$ for CIV and $53 \mathrm{~m} \AA$ for $\mathrm{MgII})$ and the small CCD arrays for the scientific cameras, the CIV alignment must be within $0.1 \mathrm{~nm}$ and the MgII alignment within $0.2 \mathrm{~nm}$ (Figure 9 and 10). In order to achieve that accuracy a platinum source was used to calibrate the wavelength drive of the Acton VM504 monochromator. The correlation between the spectral measurements at the exit of the VM504 and the National Institute Standards and Technology (NIST) platinum data binned to the same spectral resolution (http://physics.nist.gov/PhysRefData/platinum/contents.html) gives an uncertainty in the wavelength position for the monochromator of $0.1 \mathrm{~nm}$. With the entrance and exit slits of the monochromator set to 10 um, the wavelength resolution of the VM504 is quoted to be $\sim 0.06 \mathrm{~nm}$ at $436 \mathrm{~nm}$ with a position accuracy at $500 \mathrm{~nm}$ of $\pm 0.2 \mathrm{~nm}$ and a reproducibility of $\pm 0.05 \mathrm{~nm}$. Figure 11 and 12 compares the NIST platinum source data with the measurements made with the VUV collimator. Although the position error is within the quoted resolution, improving the wavelength resolution of the platinum source may require mechanical adjustments between the source and the VM504 grating. The wavelength resolution with our current setup appears to be limited to $1.5 \AA$ (Figures 11 and 12). 
Polarization measurements of the individual components have been reported elsewhere $8,9,10,11,12$. Ideally, an end to end polarization test should be made as a final system check. Unfortunately, providing a collimated beam of a known polarization over the $30 \mathrm{~cm}$ aperture of SUMI in the UV is difficult. Therefore, the instrumental polarization from the VM504 and Newtonian telescope will be monitored by the polarization optics that were used to test the SUMI optics at the NSSTC UV calibration facility ${ }^{11}$ and this instrumental polarization will be used as the source for the SUMI polarization tests.

\section{SUMMARY}

All of the optical components have been measured and meet the instrument requirements. The major mechanical components have been designed and fabricated and the payload is being assembled. Although some optical testing has been delayed due to reduce funding, the development of the VUV collimator and its polarization testing should be completed by October 1, 2007. Along with the completion of the VUV collimator, all of the optical components are expected to be epoxied into the mechanical structure. Therefore, with the FY2008 funding, the final system alignment and vibration tests are expected to be completed next spring with a launch date set for the June 2008.

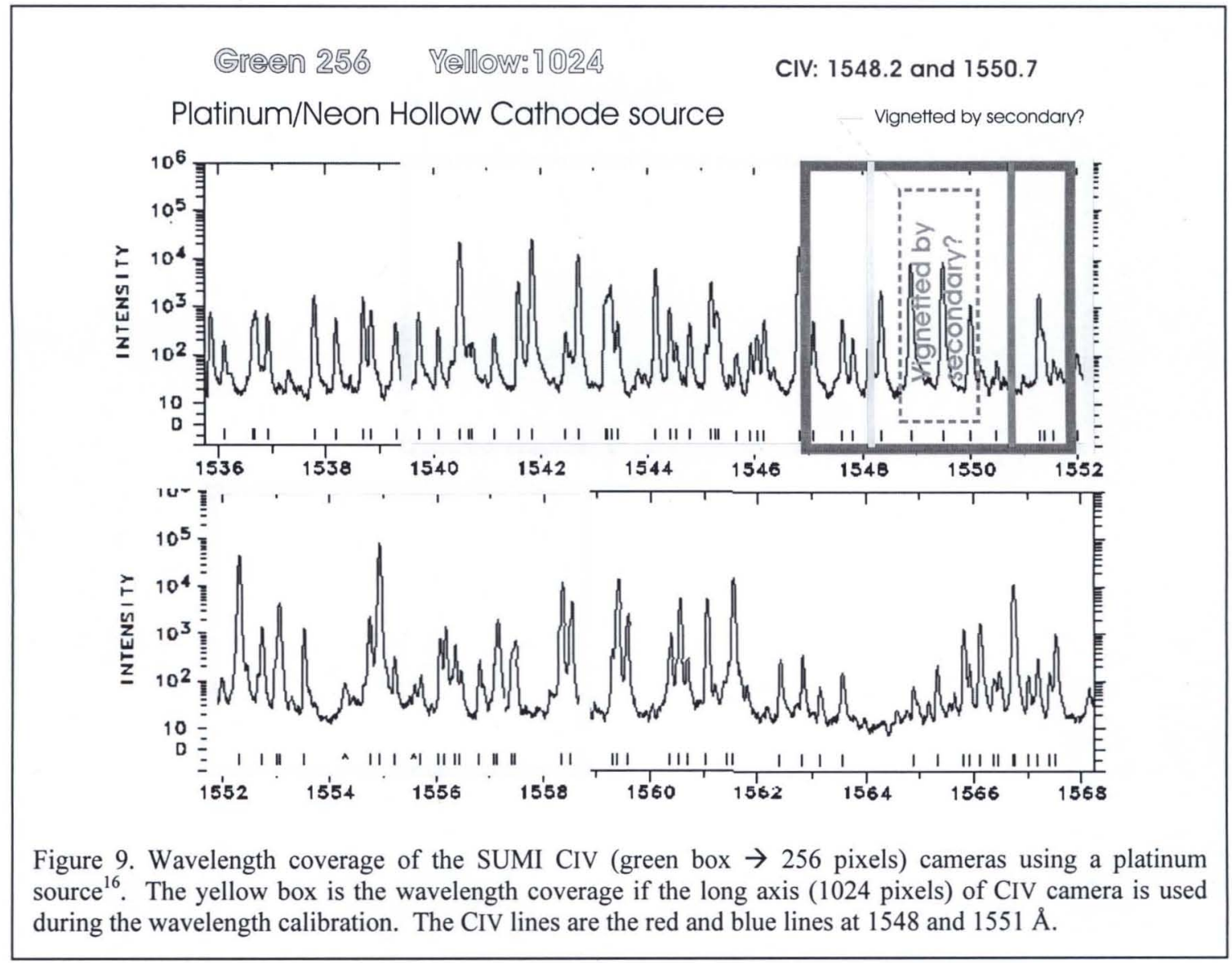




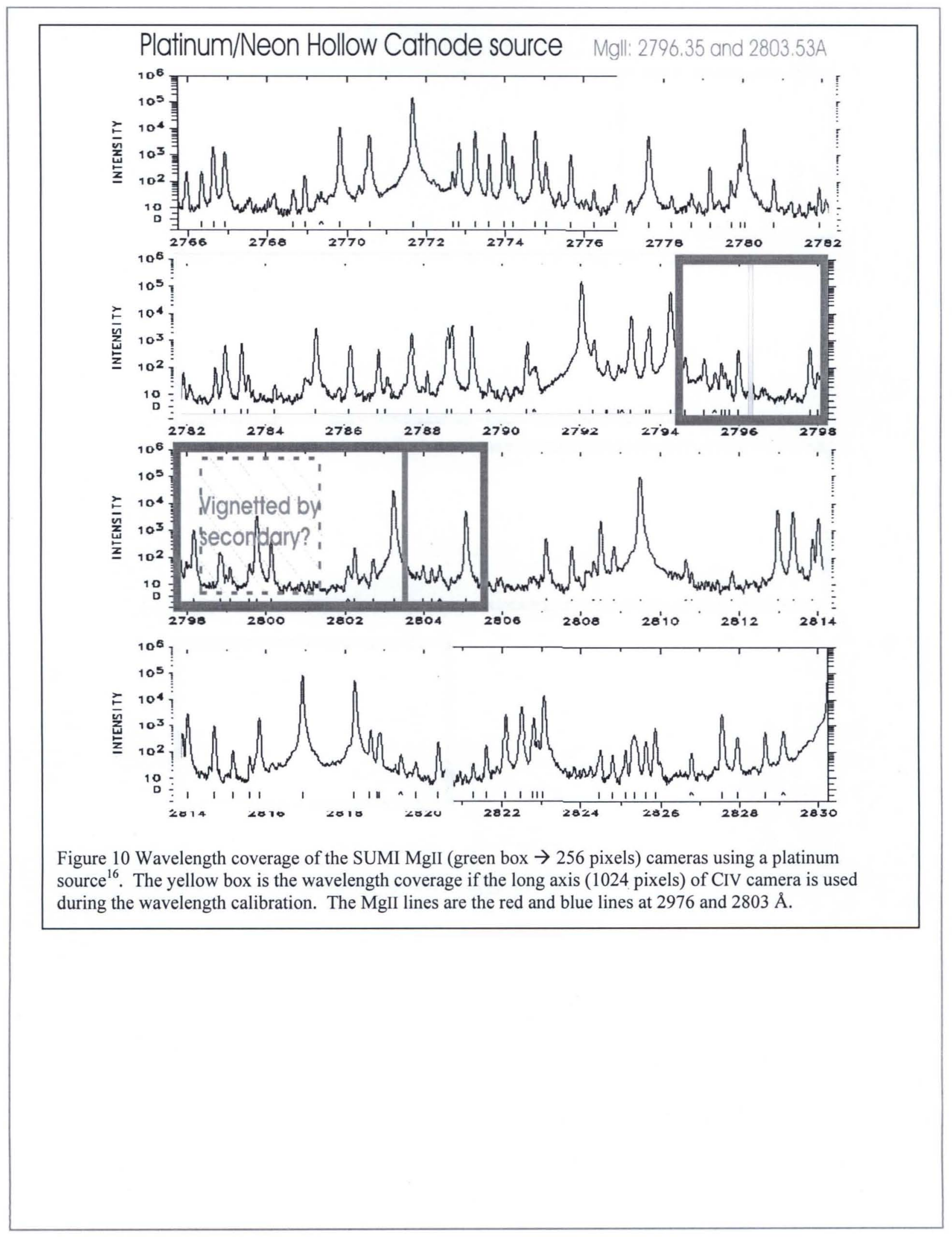




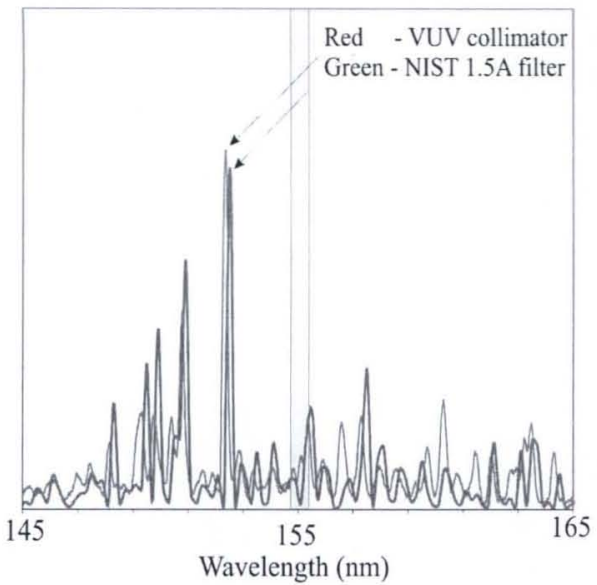

Figure 11. Comparison of VUV Platinum source measurements with NIST $^{16}$ data using a $1.5 \AA$ filter

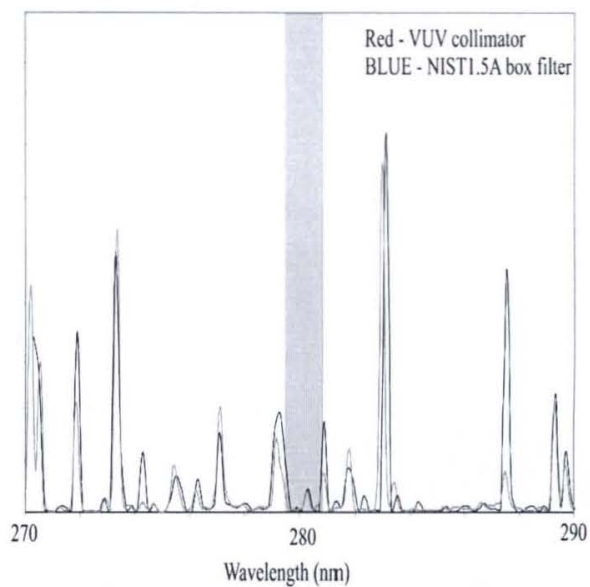

Figure 12. Comparison of VUV Platinum source measurements with NIST $^{16}$ data using a $1.5 \AA$ filter

\section{REFERENCES}

1. H. Uitenbroek, "Multilevel Radiative Transfer with Partial Frequency Redistribution," Astrophysical Journal, 557, 389-398, 2001.

2. H. Peter, "On the nature of the transition region from the chromosphere to the corona of the Sun," $A \& A, \mathbf{3 7 4}, 1108$ $1120,2001$.

3. G. A. Gary, "Plasma Beta above a Solar Active Region: Rethinking the Paradigm," Solar Phys., 203, 71, 2001.

4. R. Rosner, B. C. Low, and T. E. Holzer, "Physical processes in the solar corona," Physics of the sun, D. Reidel Publishing Co, 135-180, 1986

5. Moore, Davis, and Hathaway, "High-Resolution Solar Magnetography from Space: Beyond Solar-B," http://solarscience.msfc.nasa.gov/Beyond_Solar-B.shtml, 2001

6. J. Davis, E. West, R. Moore, G. Gary K. Kobayashi, J. Oberright, D. Evans, H. Wood, J. L. R. Saba and D. Alexander, "MTRAP: The Magnetic Transition Region Probe," Solar Physics and Space Weather Instrumentation, Proc. SPIE, 5901, p. 590112-1 to -8, 2005.

7. Gurman, J. B., "The MG II H line in sunspot umbrae," Solar Phys., 90, 13-15, 1984.

8. West, E. A., J. G. Porter, J. M. Davis, G. A. Gary, D. M. Rabin, R. J. Thomas, and J. M. Davila, "Overview of the Solar Ultraviolet Magnetograph Investigation," SPIE,Vol. 4139, pp. 350-361 (2000).

9. West, E. A., J. G. Porter, J. M. Davis, G. A. Gary, and M. Adams, "Development of a polarimeter for magnetic field measurements in the ultraviolet," SPIE Vol. 4481, pp. 109-117 (2001).

10. West, E. A., J. G. Porter, J. M. Davis, G. A. Gary, M. Adams, S. Smith and J. F. Hraba, "Optical characteristics of the Marshall Space Flight Center Solar Ultraviolet Magnetograph," SPIE Vol. 4498, pp. 101-110 (2001).

11. K. Kobayashi, E. A. West and M. Noble, "Polarization measurements in the Vacuum Ultraviolet," Polarization Science and Remote Sensing II, Proc SPIE, 5888, p. 58880G-1 to -12, 2005.

12. K. Kobayashi, E. A. West, J. M. Davis and G. A. Gary, "Polarization measurements on SUMI's TVLS gratings", Polarization Science and Remote Sensing III, Proc SPIE 6682-32, 2007.

13. T. Kita and T. Harada, "Use of Aberration-Corrected Concave Gratings in Optical Demultiplexers," Applied Optics, 22, 819-825, 1983.

14. R. J. Thomas, "Toroidal varied line-space (TVLS) gratings," Proc SPIE, 4853, 411-418, 2003.

15. L. Poletto and R. J. Thomas, "Stigmatic Spectrometers for Extended Sources: Design with Toroidal Varied LineSpace (TVLS) Gratings," Applied Optics, 43, 2029-2038, 2004.

16. NIST platinum source data at http://physics.nist.gov/PhysRefData/platinum/contents.html. 